\title{
Fabrication of optical nanodevices through field-emission scanning probe lithography and cryogenic etching
}

Cemal Aydogan, Martin Hofmann, Claudia Lenk, Burkhard Volland, Ivo W. Rangelow, et al.

Cemal Aydogan, Martin Hofmann, Claudia Lenk, Burkhard Volland, Ivo W. Rangelow, Mahmut Bicer, B. Erdem Alaca, Onur Ates, Hamdi Torun, Arda D. Yalcinkaya, "Fabrication of optical nanodevices through field-emission scanning probe lithography and cryogenic etching," Proc. SPIE 10584, Novel Patterning Technologies 2018, 105841G (19 March 2018); doi: 10.1117/12.2305268 


\title{
Fabrication of optical nanodevices through field-emission scanning probe lithography and cryogenic etching
}

\author{
Cemal Aydogan ${ }^{1,2}$ \\ ${ }^{1}$ Electronics and Cryptology Department TUBITAK - YITAL, Gebze, Kocaeli, Turkey
}

\author{
Martin Hofmann ${ }^{2}$, Claudia Lenk ${ }^{2}$, Burkhard Volland ${ }^{2}$, Ivo W. Rangelow ${ }^{2}$ \\ ${ }^{2}$ Department of Micro- und Nanoelectronic Systems, Institute of Micro- and Nanoelectronics, \\ Faculty of Electrical Engineering and Information Technology, Ilmenau University of \\ Technology, Gustav-Kirchhoff-Straße 1, 98693 Ilmenau, Deutschland \\ Mahmut Bicer ${ }^{3}$, B. Erdem Alaca ${ }^{3,4}$ \\ ${ }^{3}$ Department of Mechanical Engineering, Koc University, Rumelifeneri Yolu, 34450 Sariyer, \\ Istanbul, Turkey \\ ${ }^{4}$ Koc University Surface Science and Technology Center (KUYTAM), Rumelifeneri Yolu, \\ 34450 Sariyer, Istanbul, Turkey \\ Onur Ates ${ }^{5}$, Hamdi Torun ${ }^{5}$, Arda D. Yalcinkaya ${ }^{5}$ \\ ${ }^{5}$ Department of Electrical and Electronics Engineering, Bogazici University, 34342 Bebek, \\ Istanbul, Turkey
}

\begin{abstract}
Sub-10 nanometer lithography is opening a new area for beyond-CMOS devices. Regarding to single nano-digit manufacturing we have established a new maskless patterning scheme by using field-emission, current controlled Scanning Probe Lithography (cc-SPL) in order to create optical nanodevices in thin silicon-oninsulator (SOI) substrates. This work aims to manufacture split ring resonators into calixarene resist by using SPL, while plasma etching at cryogenic temperatures is applied for an efficient pattern transfer into the underlying Si layer. Such electromagnetic resonators take the form of a ring with a narrow gap, whose 2D array was the first left-handed material tailored to demonstrate the so-called left-hand behavior of the wave propagation. It is shown that the resonance frequency can be tuned with the feature size of the resonator, and the resonance frequency can be shifted further into near infrared or even visible light regions.
\end{abstract}

Keywords: nanofabrication, scanning probe lithography, cryogenic plasma etching, single nanometer manufacturing, split ring resonators. 


\section{INTRODUCTION}

Scaling down of device sizes has been the fundamental strategy for improving the performance and efficiency of nano electronic-mechanical devices. Field-Emission Scanning Probe Lithography (FE-SPL) permits a more precise and cost effective fabrication route towards new-generation devices. An intense electric field causes a Fowler-Nordheim-type electron emission of low energy electrons from a sharp scanning tip. Closed loop lithography offers single-nanometer manufacturing and has the potential to replace conventional lithography techniques in terms of resolution, patterning with low electron energy, direct patterning, and tone switching without any post-proccessing ${ }^{1,2}$. As opposed to Electron Beam Lithography (EBL) technique, no vacuum or special gaseous environments are required for SPL patterning. While high-energy electrons are employed to generate secondary electrons inducing the lithographic reaction in EBL, in FE-SPL we directly generate "lithographically usable" electrons in an energy range (typically $<50 \mathrm{eV}$ ) just needed for the chemical interaction with the resist. The tone of patterning can be switched by varying the exposure dose of electrons ${ }^{4}$, i.e. a wet development step is not required for obtaining positive tone lithography ${ }^{3,4}$.

AFM-imaging directly after exposure can be obtained by using the same active cantilever in imaging mode and it allows the inspection of the generated features by a closed loop imaging scheme. Moreover, using a relatively thin resist layer of calixarene, (usually with thicknesses of 10 to $50 \mathrm{~nm}$ ), we developed a reliable dry etching recipe operating at cryogenic temperatures. The process is established for linewidths below $10 \mathrm{~nm}$ and for HAR in the 50-100nm regime, respectively. As feature sizes shrink, approaching the $5 \mathrm{~nm}$ regime, feature size and the shapes must be controlled with tolerances approaching the sub-nanometer scale.

EBL based cryoetching is a well-known technique ${ }^{5,6,7}$. In order to transfer sub-10 nm structures generated by FE-SPL, an Oxford Instruments Cobra 100 ICP etcher was used. To achieve a good thermal contact between substrate and electrode, helium backside cooling was employed. To generate a DC bias, the bottom electrode was powered by an RF frequency of $13.56 \mathrm{MHz}$. The process pressure is kept under $6 \mathrm{mTorr}^{8}$.

In this work we present the manufacturing of sub-10nm half-pitch split ring resonators and the simulation results obtained for their expected behavior.

\section{FIELD-EMISSION SCANNING PROBE LITHOGRAPHY}

The capability of patterning single nanometer structures is opening a new horizon for future nanoelectronics. Theoretically, scanning probes are able to image and pattern structures down to the atomic scale ${ }^{9,10}$. In this case, quantum devices, i.e. single-electron devices working at room-temperature, could be manufactured ${ }^{33}$. We have developed a scanning probe lithography by using field-emission (FE-SPL) on molecular glass resist calixarene ${ }^{1}$. Applying a bias voltage between tip and sample results in a current flow under ambient conditions. This constant Fowler-Nordheim electron emission causes direct patterning on the resist coated sample. These low energy electrons are regulated by the current feedback loop of the SPL system. A closed loop lithography scheme enables the imaging of the patterned structure after SPL with the same cantilever and tools. 

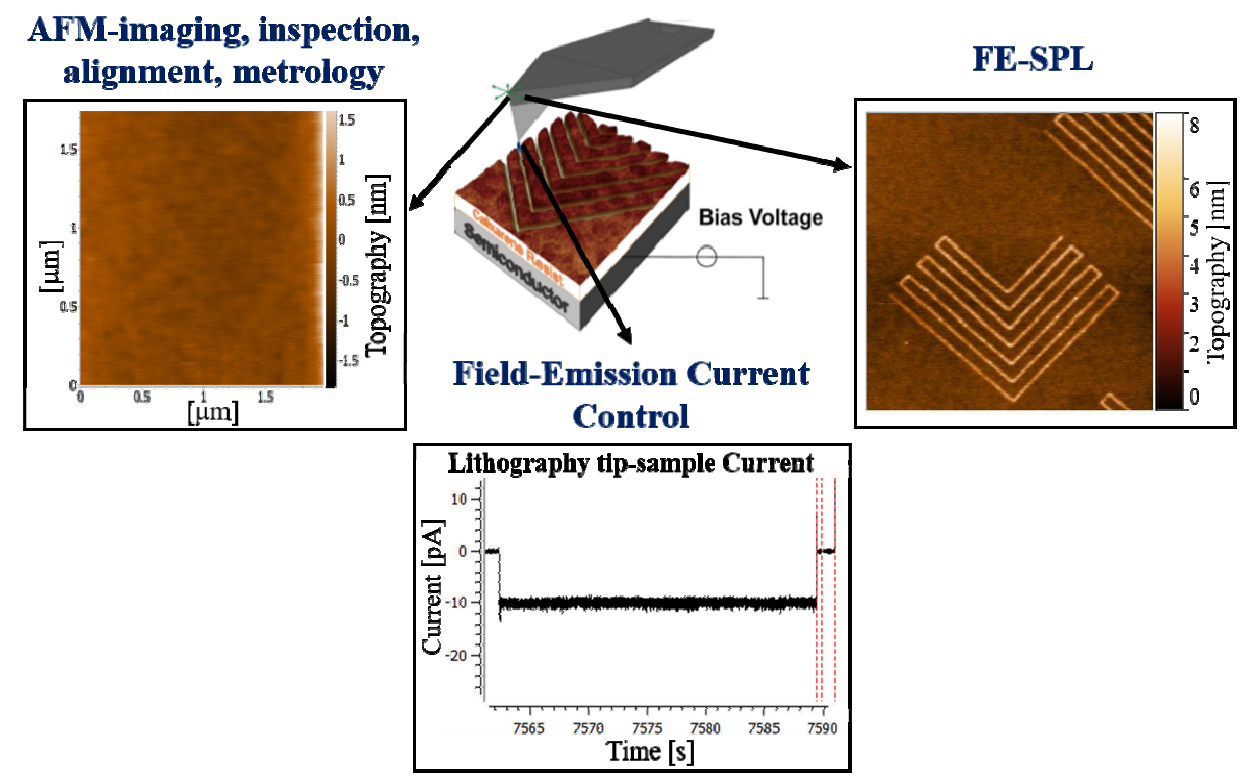

Figure 1. Concept of closed-loop operation for imaging and patterning ${ }^{2}$. The same tip that is employed for patterning via low-energy electron emission is used for imaging. This approach also facilitates pattern overlay alignment.

By varying the exposure dose through the applied current, bias voltage and the speed of patterning, we are able to crosslink the resist (negative tone) or to remove it directly (positive tone patterning) by applying higher exposure doses. No further chemical processing is required to switch the tone from negative to positive and vice versa $^{11}$. Unlike electron beam lithography, the structure is patterned directly with low energy electrons $(<50 \mathrm{eV})$ in FE-SPL. Thus, SPL is more efficient and works practically without proximity effects.

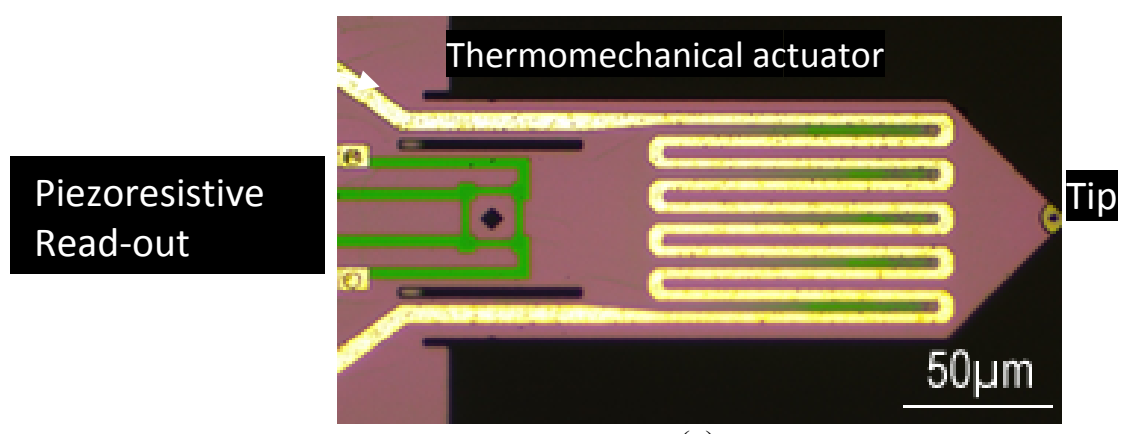

(a)

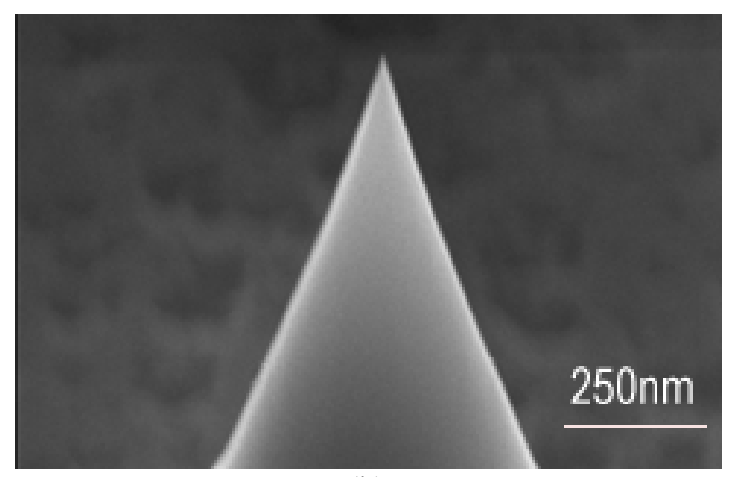

(b)

Figure 2. (a) SEM image of the thermomechanically actuated piezoresistive cantilever with piezoresistive readout. (b) A sub-10nm write and imaging resolution by SPL/AFM requires very sharp cantilever tips ${ }^{12}$. 
In our FE-SPL tool two closed loops are used; one of them is responsible for patterning through electric fieldcurrent control, while the second one is required for imaging by a force feedback. Fig. 1 shows the concept of closed-loop operation for imaging and lithography. A patterned structure is imaged directly after patterning by the dynamic mode AFM. The switching from lithography mode to imaging mode is very fast thereby minimizing the mechanical drift. For the demonstration of this work, we used a self actuated, self sensing active cantilever technology with a typical $100 \mathrm{kHz}$ resonance frequency. Integrated actuators and deflection sensors on the cantilever as shown in Fig. 2 enable both high speed AFM and SPL.

A metallic microheater (colored yellow in Fig. 2(a)) forms a thermomechanical actuator for both exciting the cantilever at its resonance, and simultaneously deflecting the cantilever at lower frequencies off-resonance $(1 \mathrm{~Hz}$ to $20 \mathrm{kHz}$ ). The deflection read-out of the cantilever is realized by piezoresistive sensors integrated on the base of cantilever beam (colored green in Fig. 2(a)) ${ }^{12-16}$. Four piezoresistors are arranged in an integrated Wheatstone bridge configuration to reduce the influence of temperature variations. At the end of the cantilever beam a sharp tip with typically $<10 \mathrm{~nm}$ tip radius of curvature is integrated (Fig. 2(b)). The cantilever bends proportionally to the square of the input current by applying a DC-current to the micro-heater ${ }^{17}$. By applying the concept of closed loop lithography, a sharp tip is used for both writing and reading of features. A more detailed information of the active cantilever, their fabrication process as well as their performance is provided elsewhere ${ }^{16}$.

The general setup of FE-SPL is shown in Figure 3. The SPL system is placed on an aluminum block and damping system to minimize thermal and mechanical drift. A top scanner is mounted on a cross-beam suspension for higher mechanical stability. The sample is located on a coarse positioning bottom stage to enable an increased patterning area of up to $100 \times 100 \mathrm{~mm}^{2}$ size. Two different scanners can be applied; one providing a $10 \times 10 \mu \mathrm{m}^{2}$ scan area and another with a $100 \times 100 \mu \mathrm{m}^{2}$ scan area. However, a step-and-repeat function enables an active pattern area over 4-inch wafers ${ }^{33}$. The step-and-repeat function is mostly used to generate same structures repeatedly on the substrate. By optical navigation through a camera mounted on the top of system, structures can be found easily after etching.

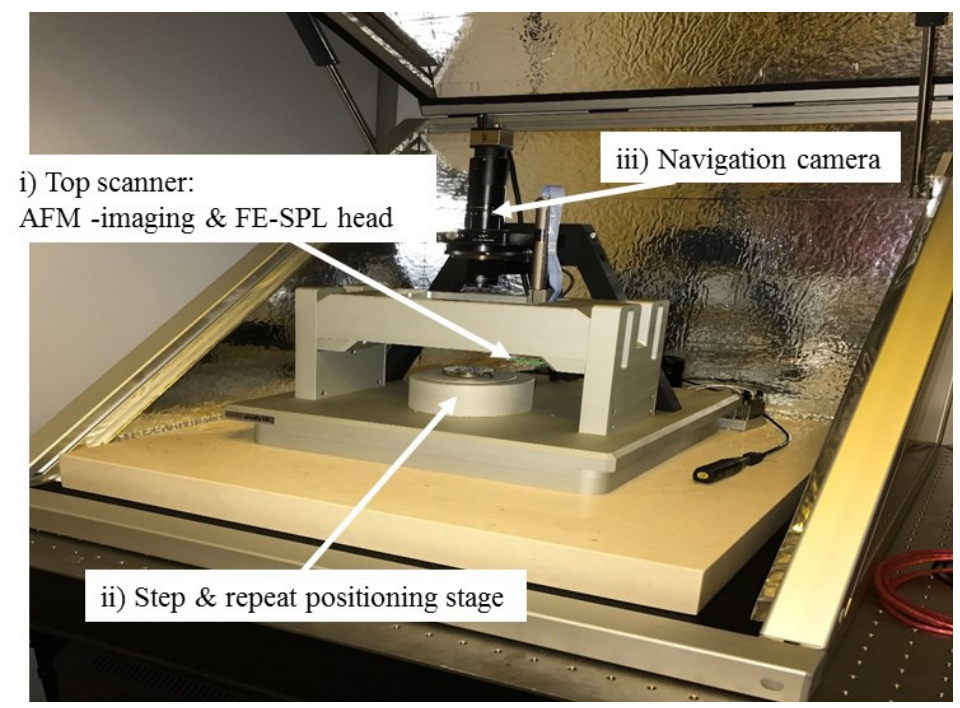

Figure 3. FE-SPL-platform from nanoanalytik GmbH with the components of i) a top scanner with AFM-imaging and FESPL head connected to a cross-beam suspension, ii) a positioning stage enabling step and repeat over a 4-inch wafer, and iii) a navigation camera. 


\section{REACTIVE ION ETCHING AT CRYOGENIC TEMPERATURE}

Cryogenic etching allows precise pattern transfer for sub $20 \mathrm{~nm}$ manufacturing with high aspect ratio. For deep silicon etching, two different dry etching methods are generally used: cryogenic processes and gas chopping processes. The cryoetching process was first introduced by Tachi et al. ${ }^{18}$, and many studies were demonstrated by a number of groups to enhance the cryoetching process ${ }^{19-23}$. The idea of cryogenic etching is to combine the ionized feed gases with low substrate temperatures typically in the range of $-100^{\circ} \mathrm{C}$ to $-140^{\circ} \mathrm{C}{ }^{18}$. The low temperature increases the sticking probabilities of the reactants ${ }^{29}$ and allows the formation of a polymeric layer on the silicon surface as shown in Fig. 4. In this work we used $\mathrm{SF}_{6} / \mathrm{O}_{2}$ gases, which are commonly employed for deep anisotropic etching of silicon. In this particular process, both oxygen and low-temperature process are necessary to produce the passivation layer at the feature sidewalls. The layer consists of siliconoxyflurid $\left(\mathrm{SiO}_{\mathrm{x}} \mathrm{F}_{\mathrm{y}}\right)$ and serves as a passivation layer that stops the etching process. At the bottom of the feature, the passivation layer is removed by the continuous ion bombardment allowing Si etching to take place ${ }^{26}$.

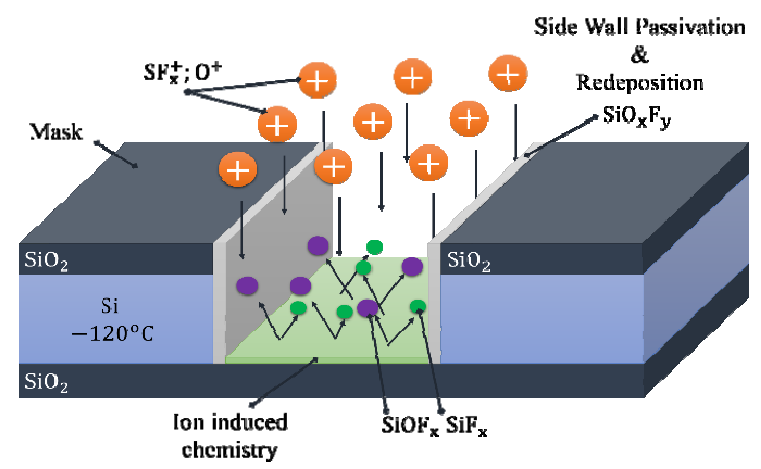

Figure 4. Demonstration of the basic principle of ions and radicals involved in cryoetching ${ }^{23,28}$.

In 2005 Mellhaoui et. al published a cryogenic etching test for different substrate temperatures, with or without oxygen and ion bombardment ${ }^{26}$. They concluded that the injection of $\mathrm{O}_{2}$ to the $\mathrm{SF}_{6}$ gas results in the formation of perfect passivation layers on the feature sidewalls whilst prevailing cryogenic temperature. At such low temperatures the reaction probability of fluorine radicals decreases and the surface coverage of the passivation precursor increases ${ }^{27-30}$. By the passivation mechanism $\mathrm{SiO}_{\mathrm{x}} \mathrm{F}_{\mathrm{y}}$ functional groups form on the sidewalls, and protect them from lateral etching. With these advantages, structures can be etched vertically. Fluorine, $\mathrm{SF}_{\mathrm{x}}$ and $\mathrm{O}$ radicals are created in the plasma source and diffuse towards the substrate. Fluorine radicals react spontaneously with silicon atoms and form $\mathrm{SiF}_{\mathrm{x}}$ molecules at the surface. $\mathrm{SiF}_{4}$ is created via an exothermal chemical reaction and then desorbs from the surface ${ }^{32}$. While the volatility of the reaction products decreases, etching occurs only in ion bombardment direction ${ }^{31}$.

For our purposes cryogenic etching was carried out in an inductively coupled plasma (ICP) source with a liquid nitrogen cooled stage in an Oxford Instruments Plasma Pro System 100 Cobra equipment. The tool is an ICP cryoetching tool with a $3 \mathrm{~kW} / 2 \mathrm{MHz}$ ICP source and a $300 \mathrm{~W} / 13.56 \mathrm{MHz}$ platen power generator, both having automatic impedance matching. For thermal contact, the system uses "helium backside cooling", where helium is introduced into the gap between the backside of the wafer and the thermally controlled platen. It is equipped with a $1600 \mathrm{l} / \mathrm{s}$ turbo pump for obtaining vacuum in the process chamber.

Etching is carried out by $\mathrm{SF}_{6}$ reactive gas with $10 \mathrm{sccm}$ gas flow. For the passivation layer, an oxygen gas flow of $2 \mathrm{sccm}$ is introduced. Process pressure is kept at $5 \mathrm{mTorr}$. A DC bias voltage at $70 \mathrm{~V}$ and RF at 11 Watt are kept constant. An inductively coupled plasma (ICP) source is used to control plasma density independent of ion flux and ion energies. The wafer is mounted to the temperature-adjusted lower electrode with helium backside cooling. The substrate temperature is kept at $-120^{\circ} \mathrm{C}$. 
First, cross lines are patterned by SPL on $15 \mathrm{~nm}$ calixarene resist coated Si sample as shown in Fig. 5. Patterning parameters are as follows: bias voltage $=-20 \mathrm{~V}$, current $=15 \mathrm{pA}$ and tip velocity $=2 \mathrm{~cm} / \mathrm{s}$. The structures were etched with the above defined recipe. 60-nm deep etching was measured after resist striping via Oxygen Plasma. The selectivity is about 1:4. More information about cryogenic etching with SPL can be found in our previous work ${ }^{33}$.

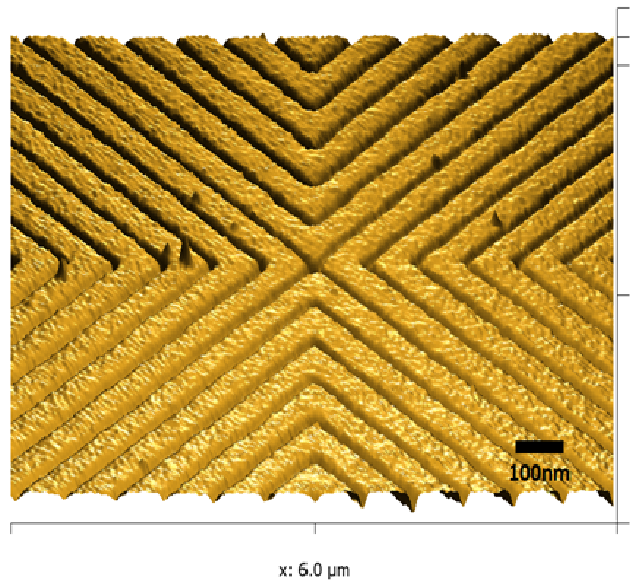

(a)

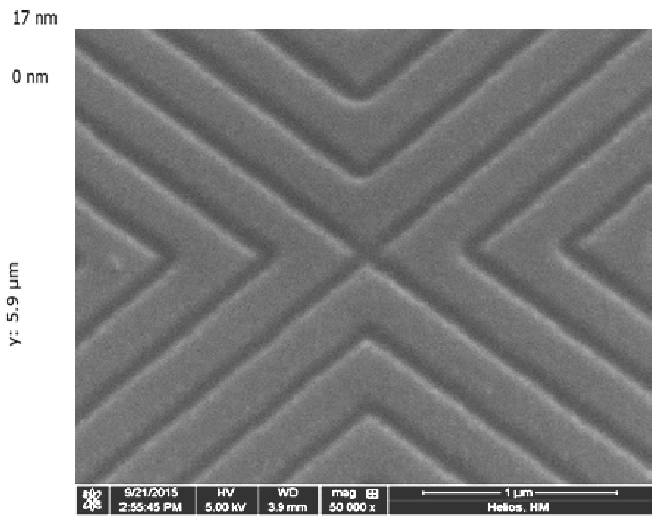

(b)

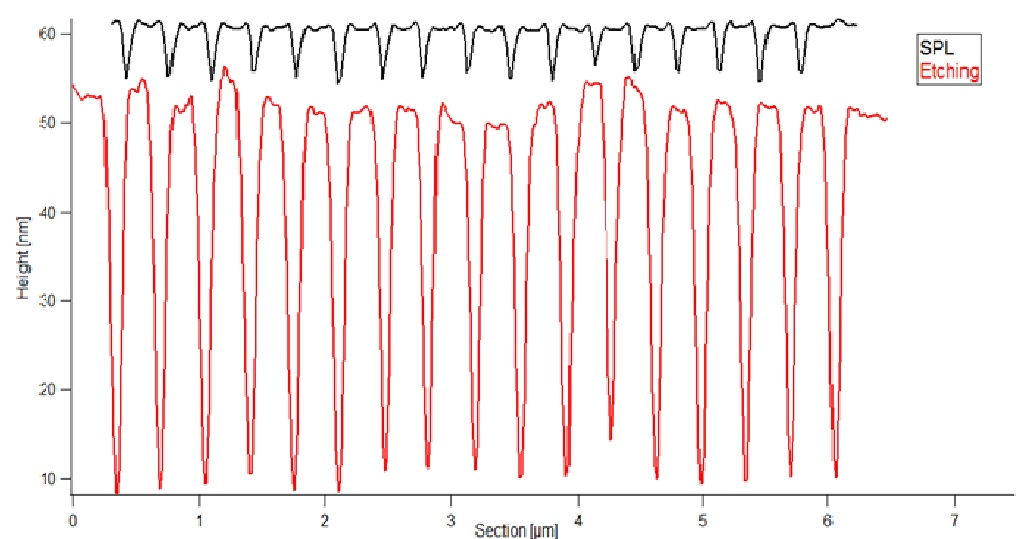

(c)

Figure 5. AFM topography images showing (a) patterned structure on $15 \mathrm{~nm}$ calixarene resist with 50nm line width, (b) the structures are etched 60nm deep at cryogenic temperature (SEM image) (c) profile of the patterns after FE-SPL and RIE at cryogenic temperature.

\section{NANOFABRICATION OF SPLIT RING RESONATORS}

Extraordinary electromagnetic behavior including negative refraction is demonstrated by metamaterials consisting of artificial lattices with a periodicity less than the wavelength of the particular radiation to be manipulated. One of the leading structures used for this purpose is the split ring resonator, a ring with a narrow gap, whose 2D array was the first left-handed material tailored to demonstrate so-called left-hand behavior of the wave propagation. In this study we demonstrate the fabrication of an array of split ring resonators within a 14-nm-thin SOI substrate with the purpose of enhancement of infrared absorption. To our knowledge this constitutes the thinnest split ring resonator architecture made of silicon reported so far.

Fabrication starts with the surface preparation of the SOI wafer, where the device layer is p-doped with an implantation dose of $10^{15} \mathrm{~cm}^{-2}$. This is followed by spin-coating with a 12-nm-thick calixarene resist. The 
exposure is carried out in the SPL tool by nano analytik GmbH. For this purpose, a SPL tip with a resonance frequency of $82.6 \mathrm{kHz}$ is used. Cryogenic etching is carried out as outlined before.

An array of 50 by 50 split ring resonators (SRRs) are fabricated. Resulting structures have linewidths of $40 \mathrm{~nm}$, gaps of $60 \mathrm{~nm}$ and a periodicity of $2.5 \mu \mathrm{m}$ in both dimensions as depicted in Fig. 6. Cryogenic etching is observed to successfully eliminate any undercut. Hence, the linewidth for the resonators is preserved.

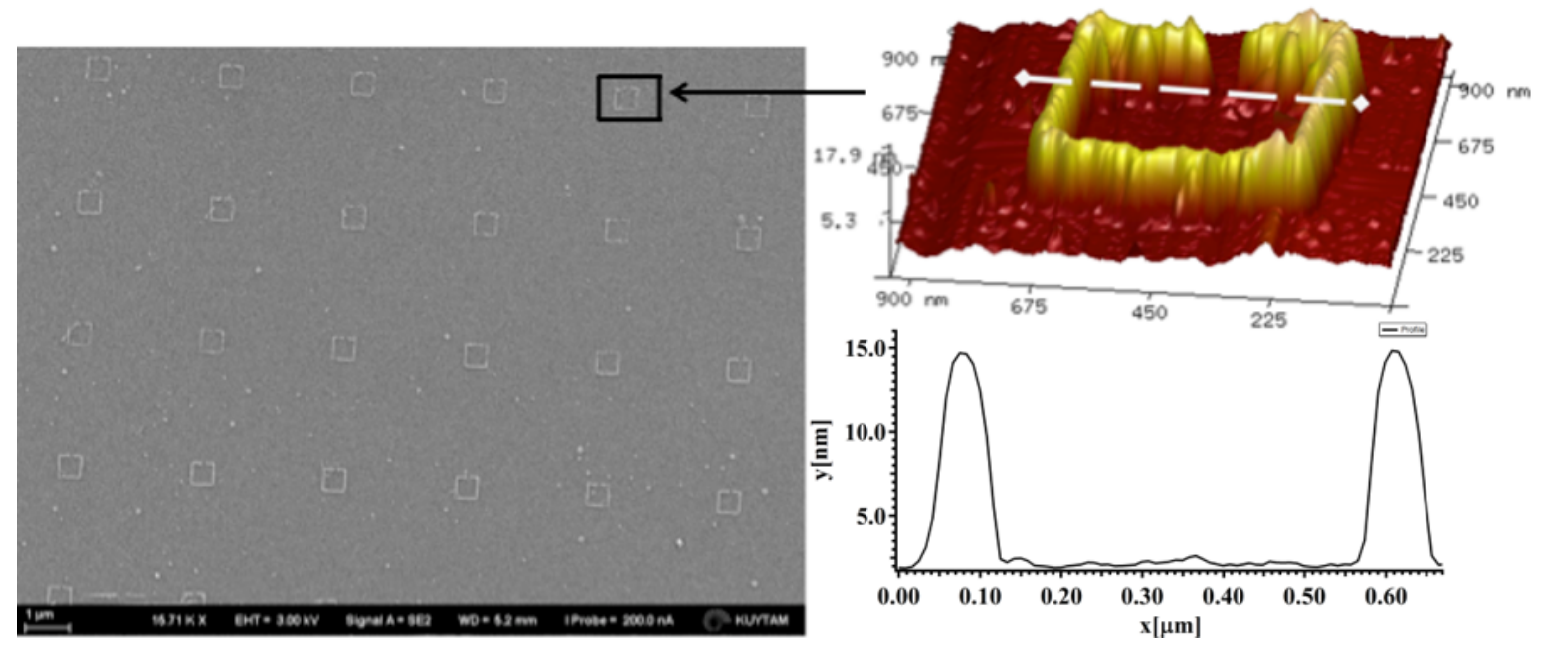

Figure 6. An array of split ring nanoresonators obtained in 14-nm-thick Si with the dimensional details of single resonators.

Considering the geometry of the devices, it is expected that such an array will exhibit geometry-controlled resonance peaks in the reflection and transmission characteristics at infrared wavelengths, which could potentially be used as a frequency selective absorber. Simulations for fabricated SRR structure are carried out by CST Microwave Studio and gold is used as device material instead of silicon to get an educated guess about the frequencies at which resonance behavior appears. The resonator in Fig. $7 \mathrm{a}$ has a linewidth of $40 \mathrm{~nm}$, a gap width of $60 \mathrm{~nm}$ and a gold thickness of $14 \mathrm{~nm}$. The electrical and optical properties of the SOI substrate are also taken into consideration in the simulation environment. The electromagnetic excitations are applied from the ports shown in Fig. $7 \mathrm{~b}$ and $7 \mathrm{c}$.

In Fig $7 \mathrm{~d}$ and $7 \mathrm{~g}$, different E-field orientations with respect to the resonator gap are depicted. In Mode 1 case, the E-field is perpendicular to the gap, while in Mode 2 the E-field is parallel to the gap. The S-parameter simulation results for Mode 1 and Mode 2 can be seen in Fig. 7e and Fig. 7h, respectively. The simulation results show that, the SRR structure demonstrates a strong absorption behavior around $2 \mu \mathrm{m}$ and $6 \mu \mathrm{m}$ wavelengths for different electric field excitations. The dips in the transmission frequency spectra are due to interaction of incident electric $(\mathrm{E})$ and magnetic $(\mathrm{H})$ fields with the SRR structure. The surface current distributions in Fig. 7f, 7i and 7j indicate different resonant modes of the SRR operation. In Fig. 7i, at resonance frequency, the incoming E-field, which is parallel to SRR gap, induces a circulating current on the SRR surface. The circulating current creates a magnetic field in the opposite direction to the incoming H-field and causes a magnetc resonance and negative permittivity at this resonance frequency. In Fig. $7 \mathrm{f}$ and $7 \mathrm{j}$, the surface current distributions indicate that the incoming E- field creates a localized potential difference on the SRR surface and splits the device into two potentially different regions. The potential difference on the SRR surface creates electric dipole moment and causes a purely electric resonance characteristic, which is always at higher frequencies. As indicated before, the resonance frequency can be tuned with the feature size of the resonator, and the resonance frequency can be shifted further into near infrared or even visible light regions. Currently, the SPL technique described in this paper can satisfy all geometric requirements that an SRR working in visible light region desires. 


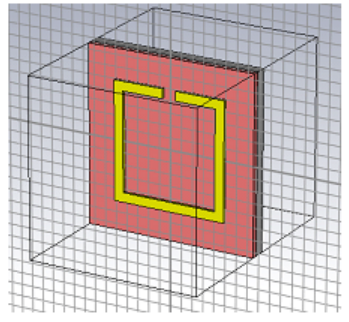

(a)

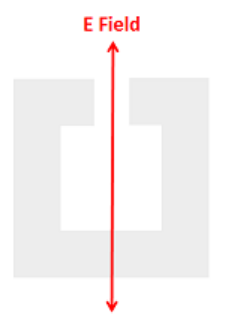

(d)

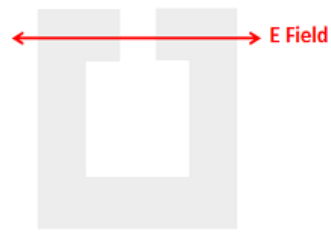

(g)

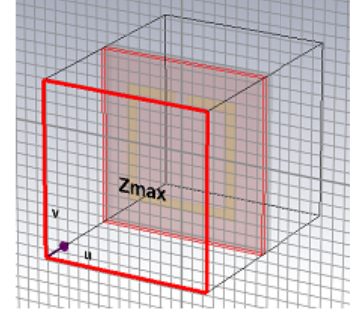

(b)

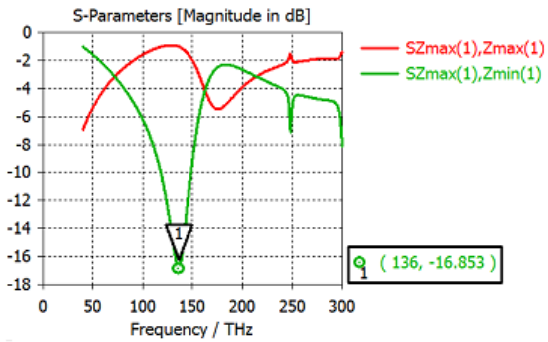

(e)

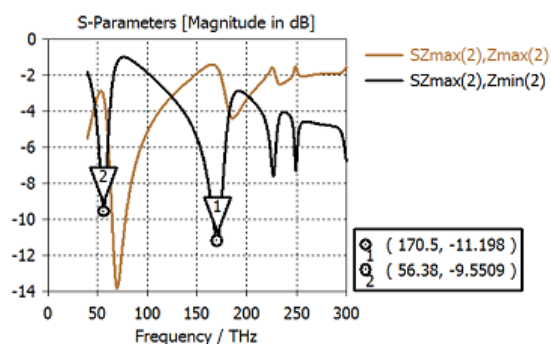

(h)

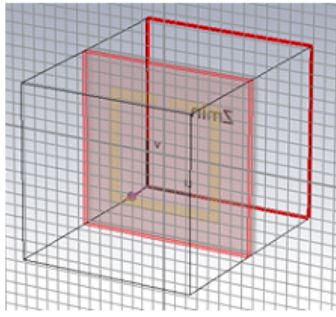

(c)

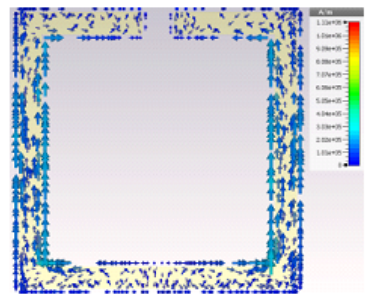

(f)

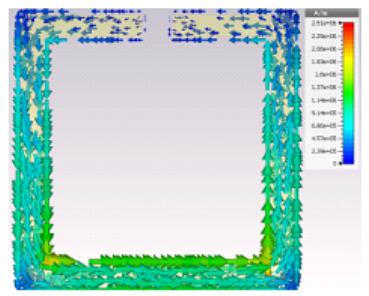

(i)

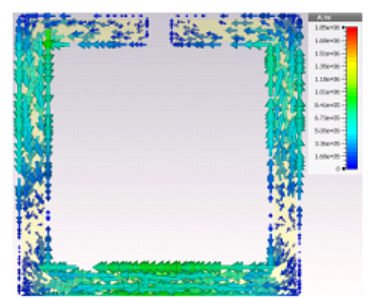

(j)

Figure 7. CST simulation results for the fabricated SRR devices. (a) Simulation setup for the SRR structure. (b-c) Port Definitions for S parameter measurement. (d) E field is perpendicular to the SRR gap (Mode 1). (e) Reflection and transmission frequency response for Mode 1 polarization. (SZMAX1-SZMAX1 is reflected power and SZMAX1SZMIN1 is transmitted power for Mode 1 polarization) f) Surface current distribution at resonance frequency for Mode 1 polarization. (g) E field is parallel to the SRR gap (Mode 2). (h) Reflection and transmission characteristic for Mode 2 polarization. (SZMAX2-SZMAX2 is reflected power and SZMAX2-SZMIN2 is transmitted power for Mode 2 polarization) $\mathrm{i}-\mathrm{j}$ ) Surface current distributions at resonance frequencies for Mode 2 polarization.

\section{SUMMARY AND CONCLUSION}

This work presents a method to manufacture SRRs by using SPL. An array of 50 by 50 SRRs are fabricated with linewidths of $40 \mathrm{~nm}$, gaps of $60 \mathrm{~nm}$ and a periodicity of $2.5 \mu \mathrm{m}$ in both dimensions. Cryogenic etching is observed to successfully eliminate any undercut preserving the linewidth upon transfer of the pattern to SOI. As 
a result, calixarene-based molecular glass resist is observed to have a selectivity of about 4 on positive tone patterned Si sample and the selectivity on negative tone patterned SOI is about 1:1. Hence, RIE at cryogenic temperatures combined with SPL is a potential candidate for future electronics and beyond-CMOS devices. Its mask- and developmentless operation, direct writing, low-energy electron emission characteristics eliminating proximity effects open a new horizon for future single nanometer manufacturing. It is shown that for the SRRs fabricated here the resonance frequency can be tuned with the feature size of the resonator, and the resonance frequency can be shifted further into near infrared or even visible light regions contributing further to the development of next-generation optical devices.

\section{ACKNOWLEDGEMENT}

This work was supported by ISTKA under Grant TR10/16/YNY/0103 "Nanotechnology Platform for the Accessible and Sustainable Pilot Fabrication of High-Added-Value Products". We would like to thank Aziz Ulvi Caliskan for holding the TUBITAK YITAL laboratory always open.

\section{REFERENCES}

[1] Rangelow, I. W., Ivanov, T., Sarov, Y., Schuh, A., Frank, A., Hartmann, H., Zöllner, J.-P., Olynick, D. and Kalchenko, V., "Nanoprobe maskless lithography," Proc. SPIE - Int. Soc. Opt. Eng. 7637, 76370V (2010).

[2] Durrani, Z., Jones, M., Kaestner, M., Hofer, M., Guliyev, E., Ahmad, A., Ivanov, T., Zoellner, J.-P. and Rangelow, I.W., "Scanning probe lithography approach for beyond CMOS devices," Proc. SPIE - Int. Soc. Opt. Eng. 8680, 868017 (2013).

[3] Custance, O., Perez, R. and Morita, S., "Atomic force microscopy as a tool for atom manipulation," Nat. Nanotechnol. 4, 803- 810 (2009).

[4] Kaestner, M., Hofer, M. and Rangelow, I. W., "Nanolithography by scanning probes on calixarene molecular glass resist using mix-and-match lithography," J. Micro/Nanolith. MEMS MOEMS 12(3), 031111 (2013).

[5] Rangelow I.W., [Deep etching of silicon], Publisher Politechnica, ISBN83-7085-254-8, (1996).

[6] Hudek P., Rangelow I.W., [Lithography and reactive ion etching], Student Text Book, University of Kassel, ITP, (1998).

[7] Rangelow I.W., "Dry etching-based silicon micro-machining for MEMS", Vacuum, 62(2-3), 279-291 (2001).

[8] Ishchuk, V., Olynick, D.L., Liu, Z. and Rangelow, I.W., "Profile simulation model for sub-50 nm cryogenic etching of silicon using SF6/O2 inductively coupled plasma," J. Appl. Phys. 118, 053302 (2015).

[9] Eigler, D.M. and Schweizer, E.K., "Positioning single atoms with a scanning tunneling microscope," Nature 344, 524-526 (1990).

[10] Custance, O., Perez, R. and Morita, S., "Atomic force microscopy as a tool for atom manipulation," Nat. Nanotechnol. 4(12), 803-810 (2009).

[11] Kaestner, M.,"Advanced electric-field scanning probe lithography on molecular resist using active cantilever” J. Micro/Nanolith. MEMS MOEMS 14(3), 031202 (2015).

[12] Rangelow, I.W., "Scanning proximity probes for nanoscience and nanofabrication," Microelectron. Eng. 83, 1449-1455 (2006).

[13] Ivanova, K., Sarov, Y., Ivanov, T., Frank, A., Zollner, J., Bitterlich, C., Wenzel, U., Volland, B. E., Klett, S., Rangelow, I. W., Zawierucha, P., Zielony, M., Gotszalk, T., Dontzov, D., Schott, W., Nikolov, N., Zier, M., Engl, W., Sulzbach, T. and Kostic, I., "Scanning proximal probes for parallel imaging and lithography," J. Vac. Sci. Technol. B 26, 2367-2373 (2008).

[14] Ivanov, T., Gotszalk, T., Sulzbach, T. and Rangelow, I. W., "Quantum size aspects of the piezoresistive effect in ultra-thin piezoresistors," Ultramicroscopy 97, 377-384 (2003).

[15] Gotszalk, T., Grabiec, P. and Rangelow, I.W. "Piezoresistive sensors for scanning probe microscopy," Ultramicroscopy 82, 39-48 (2000). 
[16] Kaestner, M., Ivanov, T., Schuh, A., Ahmad, A., Angelov, T., Krivoshapkina, Y., Budden, M., Hofer, M., Lenk, S., Zoellner, J.-P., Rangelow, I. W., Reum, A., Guliyev, E., Holz, M. and Nikolov, N., "Scanning probes in nanostructure fabrication,“ J. Vac. Sci. Technol. B 32, 06F101 (2014).

[17] Ivanova, K., Sarov, Y., Ivanov, T., Frank, A., Zöllner, J., Bitterlich, C., Wenzel, U., Volland, B.E., Klett, S., Rangelow, I.W. and Zawierucha, P., "Scanning proximal probes for parallel imaging and lithography," J. Vac. Sci. Technol. B 26, 2367 (2008).

[18] Tachi, S., Tsujimoto, K., Arai, S. and Kure, T., "Low-temperature dry etching,” J. Vac. Sci. Technol. A 9(3), 796-803 (1991).

[19] Aydil, E.S., Gregus, J.A. and Gottscho, R.A., "Electron cyclotron resonance plasma reactor for cryogenic etching,” Rev. Sci. Instrum. 64(12), 3572-3584 (1993).

[20] Oehrlein, G.S. and Kurogi, Y., "Sidewall surface chemistry in directional etching processes," Mater. Sci. Eng. 24(4), 153-183 (1998).

[21] Aachboun, S. and Ranson, P., "Deep anisotropic etching of silicon,” J. Vac. Sci. Technol. A 17(4), 22702273 (1999).

[22] Zijlstra, T., van der Drift, E.W.J.M., De Dood, M.J.A., Snoeks, E. and Polman, A., "Fabrication of twodimensional photonic crystal wave guides for 1.5 um in silicon by deep anisotropic dry etching," J. Vac. Sci. Technol. B 17(6), 2734-2739 (1999).

[23] Rangelow I.W., "Dry etching-based silicon micro-machining for MEMS," Vacuum 62, 279-291 (2001).

[24] Walker, M.J., "Comparison of Bosch and cryogenic processes for patterning high aspect ratio features in silicon," Proc. SPIE 4407, 89-100 (2001).

[25] Dussart, R., Tillocher, T., Lefaucheux, P. and Boufnichel, M., "Plasma cryogenic etching of silicon: from the early days to today's advanced technologies, "J. Phys. D: Appl. Phys. 47(12), 123001 (2014).

[26] Mellhaoui, X., Dussart, R., Tillocher, T., Lefaucheux, P., Ranson, P., Boufnichel, M. and Overzet, L.J., “SiOxFy passivation layer in silicon cryoetching," J. Appl. Phys. 98(10), 104901 (2005).

[27] Bartha, J.W., Greschner, J., Puech, M. and Maquin, P., "Low temperature etching of Si in high-density plasma using SF6/O2," Microelectron. Eng. 27, 453 (1995).

[28] Rangelow, I.W. and Löschner, H., "Reactive ion etching for microelectrical mechanical system fabrication,” J. Vac. Sci. Technol. B 13(6), 2394-2399 (1995).

[29] Rangelow, I.W., "Critical tasks in high aspect ratio silicon dry etching," J. Vac. Sci. Technol. A 21 (4), 1550-1562 (2003).

[30] Olynick, D.L., Liddle, J.A., Harteneck, B.D., Cabrini, S. and Rangelow, I.W., "Nanoscale pattern transfer for Templates," NEMs, and Nano-optics, roc. of SPIE 6462, 64620J1 (2007).

[31] Craciun, G., Blauw, M.A., Van der Drift, E., Sarro, P.M. and French, P.J.,"Temperature influence on etching deep holes with SF6/O2 cryogenic plasma," J. Micromech. Microeng. 12, 390-394 (2002).

[32] Coburn, J.W. and Winters, H.F., "Plasma etching-A discussion of mechanisms," Journal of Vacuum Science and Technology, 16(2), 391-403 (1979).

[33] Rangelow, I.W., Ahmad, A., Ivanov, T., Kaestner, M., Krivoshapkina, Y., Angelov, T., Lenk, S., Lenk, C., Ishchuk, V., Hofmann, M. and Nechepurenko, D., "Pattern-generation and pattern-transfer for single-digit nano devices," JVST B, Nanotechnology and Microelectronics: Materials, Processing, Measurement and Phenomena 34(6), 06K202 (2016). 